\title{
El trabajador universitario y su salud mental desde el modelo de creencias $^{\dagger}$
}

\author{
University worker and their mental health from the belief model \\ O trabalhador universitário e sua saúde mental desde o modelo de crenças \\ Sandra Licette Padilla-Sarmiento* \\ Diana Janeth Villamizar-Carrillo** \\ Olga Mariela Mogollón-Canal***
}

\section{Resumen}

Objetivo: Analizar el estado de la salud mental del personal administrativo en el sistema de educación superior en una región de frontera, teniendo en cuenta sus creencias a partir de los procesos de susceptibilidad y motivaciones de un modelo teórico en salud. Materiales y Métodos: El enfoque metodológico utilizado fue descriptivo, exploratorio y trasversal; caracterizando los datos de una muestra conformada por 115 participantes, a quienes se les aplicó el cuestionario de evaluación integral de la salud mental para administrativos de Padilla, Mogollón y Villamizar (2015), elaborado para esta investigación desde las dimensiones del modelo de creencias y conformado por 137 ítems. La confiabilidad por alpha de Cronbrach es de 0.83. El cuestionario estuvo sujeto a juicio de expertos para la adaptación del lenguaje a la realidad colombiana. Resultados: La salud mental de los trabajadores depende de sus estados de ánimo, de los procesos comunicacionales y del manejo de la ansiedad y el estrés, convirtiéndose en barrera al no poder acceder a los servicios de salud mental si la universidad los ofreciera. Conclusiones: Para el personal administrativo su salud mental depende de las condiciones laborales que fomenten el desarrollo individual, la interacción familiar y el manejo adecuado del estrés por cargas laborales; por lo que es necesario generar políticas en comportamiento saludable.

Palabras Clave: Personal administrativo, salud mental, Educación superior, comportamiento, modelo de creencias en salud.

\section{Autor de correspondencia}

* $ه$ Psicóloga. Investigadora activa grupo Investigación Psicología y Sociedad. Docente Universidad de Pamplona. Correo: spadilla@unipamplona.edu.co. (ID) https:// orcid.org/0000-0001-9920-8132. Pamplona, Colombia.

** Psicóloga. Investigadora Grupo de Investigación Psicología y Sociedad. Docente Universidad de Pamplona. Correo: jacarrillo71@unipamplona.edu.co. (iD) https:// orcid.org/0000-0001-9180-591X. Pamplona, Colombia.

**** Psicóloga. Docente. Directora Grupo de Investigación Psicología y Sociedad. Universidad de Pamplona. Correo: olgamariela@unipamplona.edu.co. (ID) https://orcid. org/0000-0001-9546-3435. Pamplona, Colombia.

\footnotetext{
${ }^{\dagger}$ Los resultados presentados pertenecen al proyecto de investigación matriculado en la Vicerrectoría de Investigaciones de una institución de educación superior que socializa los resultados de la muestra administrativos, sin tener en cuenta la salud sexual y física que también fue explorada en docentes y estudiantes.
}

Este es un artículo bajo la licencia CC BY (https://creativecommons.org/ licenses/by/4.0/) (c) (i) 
ISSN-PRINT

1794-9831

E-ISSN 2322-7028

Vol. 15 No. 2

Jul - Dic 2018

Cúcuta, Colombia

\begin{abstract}
Objective: Analyze the mental health status of the administrative personnel of the higher education system in a border area, considering their beliefs emanating from susceptibility processes and motivations of a theoretical health model. Materials and Methods: The methodological focus used was descriptive, exploratory and cross-sectional; characterizing data from a sample composed by 115 participants, to whom the questionnaire of comprehensive evaluation for clerks of mental health was applied to in Padilla, Mogollon and Villamizar (2015), elaborated for this research from the dimensions of the beliefs model and composed of 137 items. The reliability by the Alpha Cronbach is of 0.83 . The questionnaire was subject to judgement of experts for the adaptation of language to the Colombian context. Results: The mental health of the clerk depends on their mood, communicational processes and the management of anxiety and stress, becoming an impediment not being able to access the mental health services if the university offered them. Conclusions: For the administrative personnel, their mental health depends of the work conditions that encourage individual development, family interaction and adequate management of stress caused by workload; for this, it is necessary to generate policies for a healthy behavior.
\end{abstract}

Keywords: administrative personnel, mental health, higher education, behavior, health belief model

\title{
Resumo
}

Objetivo: Analisar o estado da saúde mental do pessoal administrativo no sistema de educação superior em uma região fronteiriça, tendo em conta suas crenças a partir dos processos de susceptibilidade e motivações de um modelo teórico em saúde. Materiais e Métodos: o enfoque metodológico utilizado foi descritivo, exploratório e transversal; caracterizando os dados de uma amostra conformada por 115 participantes, a quem foi aplicado o questionário de avaliação integral da saúde mental para administrativos de Padilla, Mogollón e Villamizar (2015), elaborado para esta pesquisa desde as dimensões do modelo de crenças e conformado por 137 itens. A confiabilidade por alpha de Cronbrach é de 0,83 . O questionário esteve sujeito a juízo de expertos para a adaptação da linguagem à realidade colombiana. Resultados: A saúde mental dos trabalhadores depende de seus estados de ânimo, dos processos comunicacionais e do manejo da ansiedade e o estresse, convertendose em barreira ao não poder aceder aos serviços da saúde mental, inclusive se a universidade os oferecesse. Conclusões: para o pessoal administrativo sua saúde mental depende das condições de trabalho que fomentem o desenvolvimento individual, a interação familiar e o manejo adequado do estresse por cargas de trabalho; pelo qual é necessário gerar políticas em comportamentos saudáveis.

Palavras-chave: pessoal administrativo, saúde mental, educação superior, comportamento, modelo de crenças em saúde.

\section{Introducción}

Introducción Es importante tener en cuenta que la salud mental del trabajador se ve impactada por las relaciones en el ambiente laboral, convirtiéndose en una dimensión de fácil vulnerabilidad; su abordaje requiere tener en cuenta las formas de organización del trabajo, su extensión a los aspectos sociales y personales relacionándosele directamente con el desempeño y productividad (1).

A pesar de que el concepto de salud sea integral y comprenda las esferas física, mental y social, la primera de éstas ha sido objeto de múltiples estudios por parte de los profesionales de la salud ocupacional dejando de lado los aspectos psicológicos y sociales (2).

En cuanto a estos dos aspectos es necesario referenciar que los problemas de salud mental se estiman en una proporción significativa sobre el ausentismo laboral, ubicando su costo entre las más importantes causas de pérdida de productividad y de años de vida por incapacidad (3).

La II encuesta nacional sobre condiciones de seguridad y salud en el trabajo, teniendo en cuenta el sistema general de riesgos laborales, realizado por el Ministerio del Trabajo en el año 2013, revela que dos 
de cada tres trabajadores manifestaron estar expuestos a factores psicosociales negativos en su jornada laboral y entre un $20 \%$ y un $33 \%$ experimentaron altos niveles de estrés.

Los riesgos ergonómicos y psicosociales son identificados como prioritarios, lo que requiere se incrementen los mecanismos y acciones de promoción, prevención y control de estos factores que beneficien la salud física, mental, el bienestar y la calidad de vida laboral de los trabajadores colombianos (4).

La Organización Mundial de la Salud (OMS) estimó a principios de este siglo que cerca del $40 \%$ de los trabajadores del mundo sufren de algún tipo de trastorno mental. Por otro lado y según los datos proporcionados por el Ministerio del Trabajo en el año 2013, revelan que los trastornos mentales ocupan el tercer lugar entre las enfermedades laborales que con mayor frecuencia presentan los trabajadores.

A pesar de estas cifras, las acciones que se proponen se limitan a la intervención de los factores de riesgo psicosocial focalizados en la prevención del estrés, dejando de un lado todos los demás elementos que afectan el bienestar psicológico (5).

Desde los referentes psicológicos y teniendo en cuenta el concepto de la "universidad promotora de salud", se ha desarrollado la idea de "organizaciones saludables". Este concepto hace alusión a aquellas instituciones en las que se llevan a cabo prácticas saludables para estructurar y gestionar los procesos de trabajo; lo que permite el incremento de empleados saludables y con alto sentido de pertenencia (6).

En estos contextos, la percepción de la salud está ligada a términos como el de bienestar psicológico, dando como resultado que las personas más felices en el trabajo desarrollen una visión positiva y optimista de sus situaciones, sustentadas en la autoeficacia y la motivación (7); razón por la cual sufren menos molestias somáticas, tienen mejores apreciaciones personales, dominan el entorno y ostentan altas habilidades sociales para relacionarse con los demás y crear comunidad.

El estado de salud del personal administrativo impacta directamente sobre la economía de la institución y de la región; la OIT (Organización Internacional del trabajo) concluye que las pérdidas por enfermedad y lesiones ocupacionales representan una carga pesada para el desarrollo económico, ocasionando la pérdida del $4 \%$ del producto interno bruto (PIB) (8).

De allí la necesidad de conocer los factores de riesgo psicosociales e intervenirlos para lograr una adecuada dinámica entre el trabajo y el medio ambiente en el que se desarrolla, permitiendo de esta manera la satisfacción y las condiciones de organización positivas, incluyendo variables relacionadas con las potencialidades del trabajador y las necesidades del contexto específico; aspectos que influyen en el estado de salud y bienestar de la persona, determinando su nivel de rendimiento y la percepción de autosatisfacción.

De acuerdo con el panorama anterior y contribuyendo con este tipo de estudios, se plantea en esta investigación analizar el estado de la salud mental del personal administrativo en una institución de educación superior, teniendo en cuenta sus creencias a partir de los procesos de susceptibilidad y motivaciones enmarcadas en un modelo teórico sobre salud. Este tipo de investigación se orienta a comprender la dinámica de la salud mental, articulada al desarrollo humano evolutivo desde la diversidad de componentes (emocionales, sociales, cognitivos y espirituales) (9).

Con referencia a publicaciones relacionadas con el tema de la salud mental en educación superior, se ha encontrado que los estudiantes permiten visualizar la motivación intrínseca como un factor fundamental para la adquisición de estilos de vida saludable, siempre en función de la susceptibilidad percibida que está en directa relación con la estima de sí mismo, al igual que con su estabilidad afectiva emocional y sus hábitos (10).

De la misma forma, la comprensión de la salud mental en docentes universitarios permite establecer que éstos la perciben adaptativa, pero requieren de medios para su promoción, mitigando el riesgo en cuanto a tensiones laborales y sobrecargas de trabajo; igualmente, exigen la cooperación en la prevención y promoción para el fortalecimiento de la salud mental desde los espacios vitales de pareja, familia y el ambiente laboral (11).

El modelo de creencias es el referente teórico utilizado en esta investigación, por ser uno de los marcos conceptuales más extensamente usados para comprender el cambio y mantenimiento de comportamientos relacionados con la salud (12). 
ISSN-PRINT

1794-9831

E-ISSN 2322-7028

Vol. 15 No. 2

Jul - Dic 2018

Cúcuta, Colombia
Dentro de este marco, el comportamiento se explica teniendo en cuenta seis dimensiones: susceptibilidad, severidad, beneficios, costos o barreras percibidas, motivación y claves para la acción; variables que pueden ser cambiantes en el tiempo, siendo fundamentales la percepción de la amenaza y la intención comportamental.

Con este trabajo se pretende impactar las políticas de salud mental en las universidades, promoviendo el bienestar general y organizacional, así como el rendimiento y las relaciones interpersonales, en beneficio de la calidad de la educación que es el fin primordial del desarrollo de la sociedad (13).

\section{Objetivos}

\section{Objetivo general.}

Analizar el estado de la salud mental del personal administrativo en una institución de educación superior, teniendo en cuenta sus creencias desde los procesos de susceptibilidad y motivaciones enmarcadas en un modelo teórico en salud.

\section{Objetivos específicos.}

- Describir cada una de las seis dimensiones incluidas dentro del modelo de creencias en salud en la comunidad administrativa de una institución de educación superior.

- Identificar los elementos claves que permitan a futuro la construcción de políticas universitarias para instituciones de educación superior saludables.

\section{Materiales y Métodos}

La metodología utilizada fue descriptiva, exploratoria y trasversal. Los participantes pertenecían a las dependencias administrativas de la institución de educación superior, a quienes se les abordó en sus puestos de trabajo teniendo en cuenta la aprobación del Comité de Ética de la Universidad, aplicándose un consentimiento informado. La población total está integrada por 250 administrativos; el tamaño de la muestra fue de 115 participantes con una confiabilidad del $95 \%$, error estándar de muestreo de 0.67 y las probabilidades de selección y no selección iguales al $50 \%$. El tipo de muestreo fue aleatorio simple.

El instrumento fue elaborado por las autoras Padilla,
Mogollón y Villamizar (2015). El número total de ítems del cuestionario fue de 137, midiendo tres aspectos: salud mental (30 reactivos), salud sexual (47 reactivos), salud física (43 reactivos) y 17 preguntas sobre datos sociodemográficos. En este artículo sólo se incluyen los resultados referentes a la salud mental de los administrativos, aplicando el modelo de creencias.

La validez y confiabilidad del instrumente se obtuvo a través de una prueba piloto en una muestra con características similares, conformada por 30 personas, obteniéndose una confiabilidad a través del alpha de Cronbach de 0.83; se utilizó el programa SPSS versión 20. Igualmente se sometió al juicio de expertos para acoplar el lenguaje al contexto colombiano.

Se utilizaron diferentes escalas de Likert teniendo en cuenta las necesidades de cada dimensión Fundamentadas en la percepción. La dimensión severidad, con cinco (5) preguntas $(46,57,59,62$ y 69); susceptibilidad, consta de ocho preguntas $(44,45,47,60,67,71,72$ y 73$)$; beneficios, con 9 preguntas $(50,53,54,55,56,61,63,64$ y 70$)$; barreras, tres preguntas $(49,58$ y 65$)$; motivación para la salud, dos preguntas (48 y 52); y la dimensión claves para la acción, conformada por tres preguntas $(51,66$ y 68).

\section{Resultados}

Losresultadosestán sustentadosen elmodelodecreencias y se presentan de acuerdo a sus seis dimensiones. La muestra representativa estuvo conformada por el $53 \%$ de mujeres y $45.3 \%$ de hombres; el $1,7 \%$ no responde a la pregunta. El 80.9 \% se identifica con la religión católica como parte de sus creencias centrales, y un 19,1 $\%$ corresponde a las personas con ausencia de creencias y no sabe, no responde.

Los lugares de procedencia del personal administrativo abordado se encuentran mayoritariamente en el Norte de Santander con un 79,1\%; seguido de Santander, Bolívar, Arauca, Magdalena, César, Tolima y Guaviare.

La etapa del ciclo vital es la adultez joven y la adultez (20 - 35 años) con un aproximado del $77 \%$ de la muestra y un $23 \%$ representado en la adultez media y tardía (36 - 55 años). El estado civil de la población corresponde a: $43,5 \%$ que es soltero/a, un $28,7 \%$ que se encuentra en unión libre y/o en convivencia y el $25.2 \%$ son casados, separados o viudos. 
En cuanto a las personas con las que viven, se observó que: el $54.7 \%$ lo hace con su familia, el $25.2 \%$ con su pareja, el $17.4 \%$ vive solo, con los amigos o seleccionan respuestas diferentes y un 2.6 $\%$ no responde la pregunta. Frente al estrato socio económico los administrativos consideran que son del estrato tres un $47 \%$, un $27.8 \%$ del estrato dos, un $14.8 \%$ estrato cuatro, un $7.8 \%$ estrato uno y solo una persona cree ser estrato cinco.

El $81 \%$ de los administrativos tiene de 0 a 10 años laborando en la institución, con vinculación por contratación temporal; el $13.9 \%$ ha trabajado más de 10 años en la universidad, siendo personal de carrera.

El último nivel de estudio obtenido por el personal administrativo corresponde a un $49.5 \%$ de pregrado, un $25.2 \%$ es especialista, seguido de un $20.9 \%$ con estudios a nivel técnico, tecnológico y secundaria; otro $3.5 \%$ tiene estudios de maestría y un $0.9 \%$ no responde la pregunta.

\section{Dimensiones - Modelo de creencias en salud.}

\section{Severidad percibida.}

Teniendo en cuenta que la estructura cognitiva que tiene inmersa las percepciones individuales de lo que sucede, un $28,7 \%$, el personal administrativo cree que lo que más afecta su salud mental es la familia, seguido de los aspectos individuales con un 25,2 \% y la vida laboral con un 20,9 \% (Figura 1). En este último se incluyen los horarios, las cargas de trabajo, la relación entre los compañeros de oficina, la falta de espacios para la gimnasia laboral y la ausencia de estrategias para generar movilizaciones positivas alrededor de las situaciones diarias.

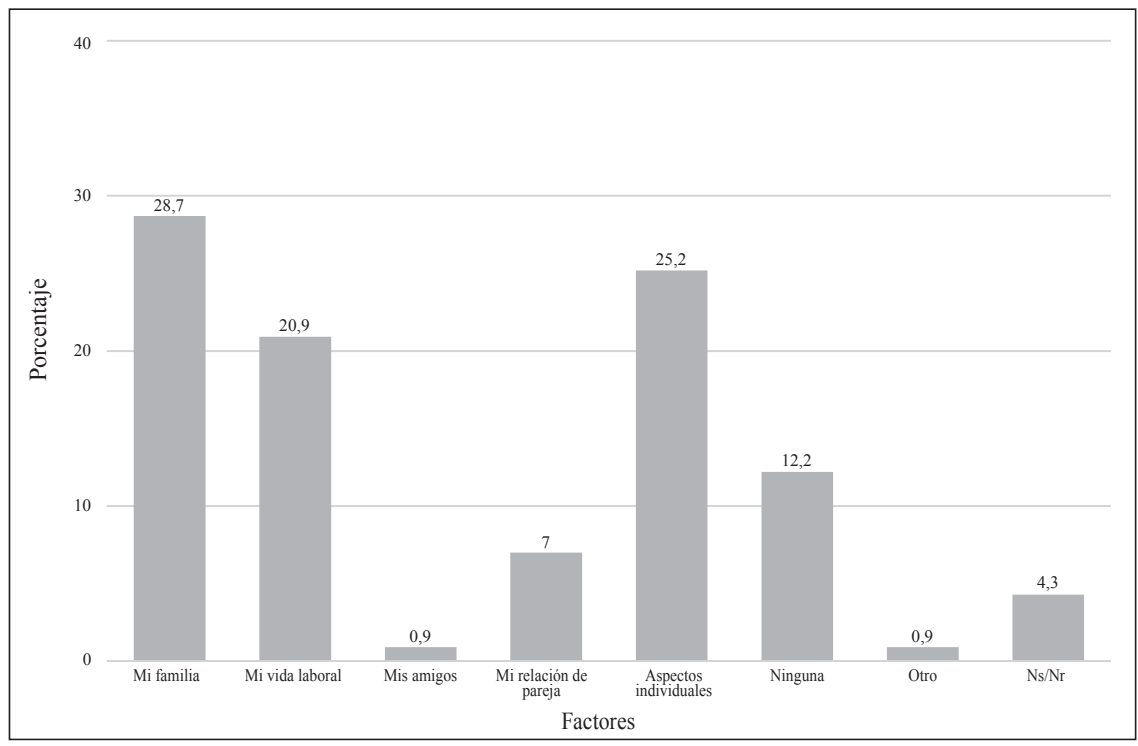

Figura 1. Análisis parcial dimensión severidad percibida personal administrativo

Fuente: Cuestionario evaluación integral de la salud mental para personal administrativo (2015)

Un menor porcentaje de la población manifiesta que ninguna de las opciones dadas afecta su salud mental y un $7 \%$ considera que la relación de pareja genera una afectación en lo que respecta al bienestar psicológico y emocional.

La población encuestada dimensiona y percibe en su gran mayoría (alrededor del $81 \%$ ) que un problema psicológico afectaría su vida cotidiana. Un aproximado del $17 \%$ de la población no sabría decir si le afectaría o no y consideran que probablemente no le perturbaría un problema psicológico su vida cotidiana.

Respecto al control de impulsos el 46,1 \% manifiesta que algunas veces lo ha perdido y solo un porcentaje menor, que corresponde a dos personas, manifiestan haberlo perdido en todas las situaciones.

Por otra parte, el 58,3\% de la población percibe que nunca ha presentado deterioro físico por problemas de salud mental; sin embargo, para un $34.7 \%$ algunas 
ISSN-PRINT

1794-9831

E-ISSN 2322-7028

Vol. 15 No. 2

Jul - Dic 2018

Cúcuta, Colombia veces han sufrido dolores de cabeza, problemas de colon, gastritis, dolor de espalda, de cuello y falta de apetito, entre otros.

Del mismo modo, la población en un 76,5 \% identifica y percibe el consumo de sustancias psicoactivas como una situación que puede generar adicción; sin embargo, un $14 \%$ aproximadamente, es indiferente frente a la situación o no responde la pregunta.

\section{Susceptibilidad Percibida.}

En general el personal administrativo considera su salud mental como el resultado de la estabilidad afectivo - emocional, la autoestima, la economía, su estado físico, así como sus hábitos y estilo de vida saludables.

La población en un $62.6 \%$ percibe que nunca ha vivido situaciones que afecten su salud mental, mientras que un $34.8 \%$ considera que algunas veces se han presentado situaciones que les han afectado y un $2.6 \%$ no responde a la pregunta.

No obstante, el $68 \%$ de los encuestados perciben una baja posibilidad de verse afectados en su salud mental. Sin embargo, el 31,3\% se ha visto afectado algunas veces y el 3,5\% percibe que casi siempre se presentan situaciones que les afecta mentalmente.

De igual manera, el $65.3 \%$ de la población considera que el estrés afecta su salud mental; un 30,4\% percibe que no y un $4.3 \%$ no responde. Respecto a los problemas que afectan la salud mental, de acuerdo a un $60 \%$ están relacionados con el manejo inadecuado del estrés; un $28.8 \%$ con cambios bruscos en el estado de ánimo, problemas en la relación de pareja, comunicación inadecuada y otros; un $11,3 \%$ no responde.

Respecto a las fortalezas el $53.1 \%$ del personal administrativo considera que la mayor fortaleza en el área de la salud mental se encuentra en los hábitos y las costumbres, en la resolución adecuada de conflictos, en la motivación, en las creencias personales y otras; entre tanto un $8,7 \%$ del personal encuestado no reconoce ninguna fortaleza y el $38.3 \%$ no responde la pregunta.

Así mismo, respecto a la mayor fortaleza de la universidad en el área de salud mental, el $38.2 \%$ de la población considera que se encuentra en la política de bienestar universitario para administrativos, la política institucional para la salud mental, la existencia de programas de promoción y la prevención; mientras que el $20.9 \%$ no identifica ninguna y el $40.9 \%$ no responde.

La Figura 2 presenta los resultados parciales obtenidos en la dimensión susceptibilidad percibida por el personal administrativo en cuanto a su salud mental.

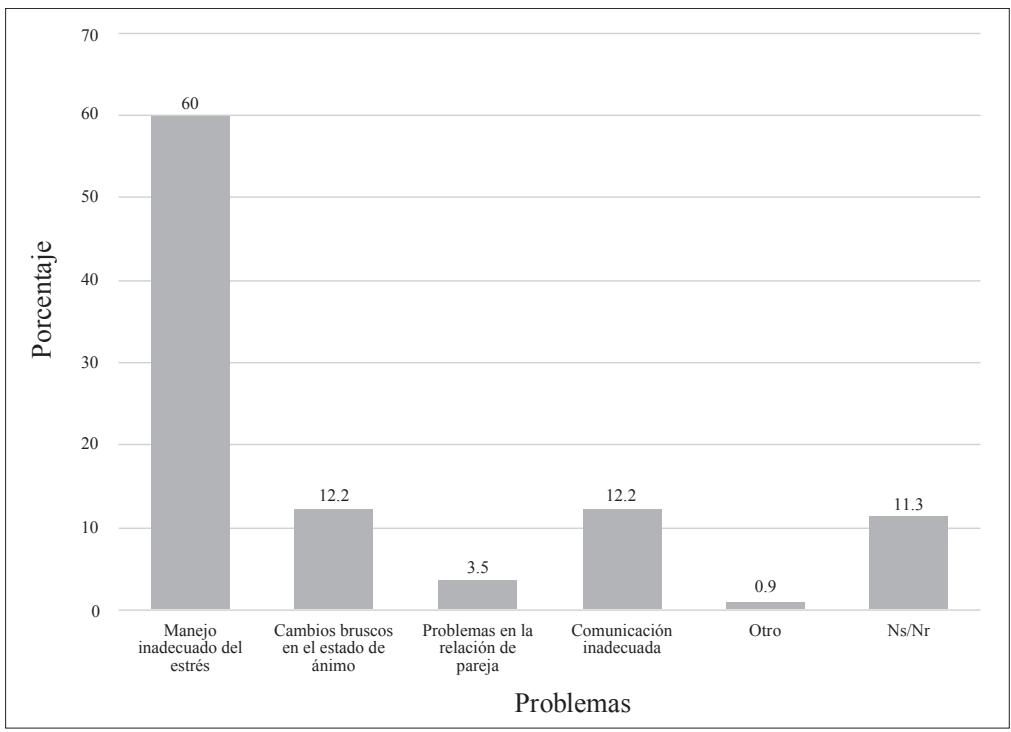

Figura 2. Análisis parcial dimensión susceptibilidad percibida personal administrativo universitario.

Fuente: Cuestionario evaluación integral de la salud mental para personal administrativo ( 2015). 


\section{Beneficios Percibidos.}

El $86.1 \%$ de la población cree en el apoyo de un profesional en salud mental y seguiría las recomendaciones realizadas por éste; el $6 \%$ del personal no está de acuerdo con ésta creencia ni con la posibilidad de seguir las recomendaciones dadas y el $7.8 \%$ no responde. No obstante, un $88.7 \%$ percibe como beneficioso un tratamiento psicológico, el 0.9 $\%$ no lo considera así y un $10.4 \%$ no responde.

Al indagar respecto al tratamiento de una enfermedad psicológica, el $93.9 \%$ de los encuestados manifestó que si se la trataría si le fuera diagnosticada; mientras que el $0.9 \%$ nunca lo haría y el $5.2 \%$ no responde. En el caso concreto en que se presente ésta enfermedad, un $92.1 \%$ del personal administrativo percibe que la relación profesional - paciente debería ser excelente, sobresaliente y aceptable; mientras que un $1.7 \%$ la considera deficiente y un $6.1 \%$ no responde.
De otra parte, el $77.4 \%$ de la población encuestada considera que nunca consume medicamentos sin prescripción médica, seguido del $18.3 \%$ que casi nunca, algunas veces y siempre lo hace, frente a un $4.3 \%$ que no responde. De igual forma, el $85.3 \%$ del personal administrativo cuenta con el apoyo emocional frente a un problema psicológico, $5.2 \%$ no lo percibe nunca y el $9.6 \%$ no responde.

La principal fuente de apoyo emocional frente a un problema psicológico lo constituye la familia en un $84,3 \%$; seguido de los amigos, compañeros de trabajo y otros con un 7,9\%; el $1.7 \%$ de la población no identifica ninguna red de apoyo y el $6.1 \%$ no responde.

De forma gráfica se muestran los beneficios percibidos por el personal administrativo en cuanto a su salud mental, en la Figura 3.

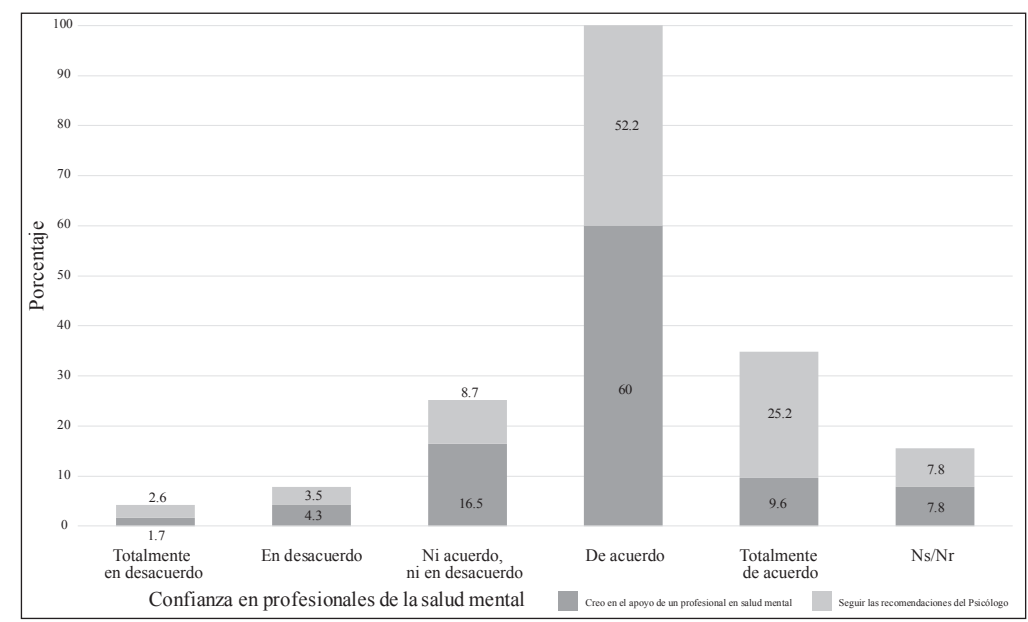

Figura 3. Análisis parcial dimensión beneficios percibidos personal administrativo universitario.

Fuente: Cuestionario evaluación integral de la salud mental para personal administrativo (2015).

\section{Barreras Percibidas}

La facilidad para acceder a un profesional de la salud mental es percibida por el $55.7 \%$ de la población encuestada, mientras que el $23.4 \%$ considera que no es fácil y un $20.9 \%$ no responde la pregunta.

Para el personal administrativo un factor positivo frente a su salud mental, es que la mayoría se siente capaz de enfrentar las situaciones difíciles que se le presentan en la vida diaria.
Sin embargo, el $65.2 \%$ de los encuestados siente que hay obstáculos para tener un estilo de vida saludable en cuanto a su salud mental, mientras que el $27.8 \%$ nunca lo percibe y el $7 \%$ no responde.

\section{Motivación para la salud.}

El $77.3 \%$ considera motivador el saber que cuenta con capacidades para disminuir el riesgo de presentar dificultades psicológicas si pone en práctica comportamientos saludables a futuro; entre tanto el 
ISSN-PRINT

1794-9831

E-ISSN 2322-7028

Vol. 15 No. 2

Jul - Dic 2018

Cúcuta, Colombia
$17.4 \%$ no lo percibe así y el $5.2 \%$ no responde la pregunta.

Las actividades que motivan al personal administrativo a practicar comportamientos saludables están relacionadas con el desarrollo: personal (autoestima, regulación afectivo - emocional y toma de decisiones) $\mathrm{y}$ social (fortalecimiento comunicativo y disfrute y bienestar).

\section{Claves para la acción.}

La población administrativa de esta Universidad en un $64.4 \%$ utilizaría estrategias para recordar los compromisos que tengan que ver con actividades relacionadas a su salud mental, el $25.2 \%$ no las aplicaría y el $10.4 \%$ no responde.

Del mismo modo, el $76.6 \%$ considera a los medios de comunicación como canales de apoyo frente a los procesos de prevención de la salud mental, mientras que el $10.4 \%$ no los considera así y el $13 \%$ no asume una postura favorable.

Igualmente, un $92.2 \%$ de la población encuestada considera el ambiente laboral como un promotor de salud mental que le permite generar claves de acción, frente al $3.4 \%$ que no lo percibe de esta forma y el $4.3 \%$ no responde.

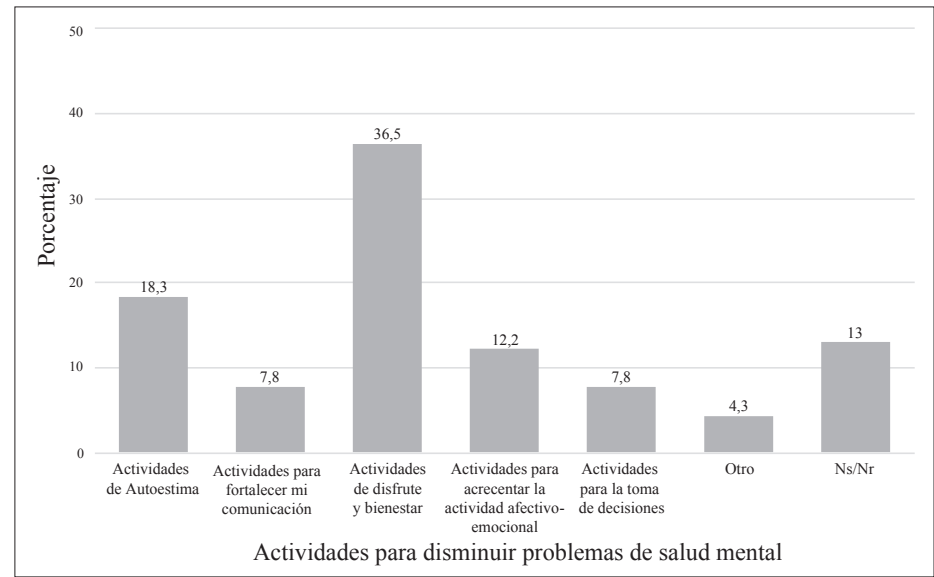

Figura 4. Análisis parcial dimensión motivación para la salud personal administrativo universitario.

Fuente: Cuestionario evaluación integral de la salud mental para personal administrativo (2015).

\section{Discusión}

Los datos sociodemográficos ubican la muestra de estudio en una región de frontera; con creencias religiosas en su mayoría pertenecientes al catolicismo con un $80.9 \%$ y al cristianismo con un $19.9 \%$; el $79.9 \%$ convive en un ambiente familiar $y / o$ de pareja; tiene facilidad de acceder a la educación superior ( $82 \%$ de la muestra cuenta con pregrado y especializaciones); lo que permite concluir que todos estos factores protectores favorecen la calidad de vida de los encuestados.

Los resultados son similares a otras investigaciones(6), donde se afirma que la espiritualidad tiene que ver con aspectos del lugar de trabajo que promueven sensaciones individuales de trascendencia y satisfacción. Además, es un factor que fortalece la vida interna de las personas, es decir que potencia la satisfacción laboral y la sensación subjetiva de salud. Altos niveles de espiritualidad permiten una percepción atenuada del estrés laboral y contribuyen al bienestar y a la salud.

Cuando existe una total cobertura en salud, situación que junto con la etapa del ciclo vital (adultez, joven e intermedia) facilitan una estructura cognitiva abierta a la educación para la salud, en un medio de modernidad y posmodernidad dentro de la política de contratación y vinculación laboral en pro de un proyecto de vida; donde el $53 \%$ de la muestra está conformada por mujeres y el $45.3 \%$ por hombres. Estos referentes también son claves para la acción, permitiendo adoptar una conducta saludable, siendo las variables demográficas (ciclo vital), socio- psicológicas y 
estructurales influyentes en las conductas de salud (14).

Algo semejante ocurre con el trabajo al ser considerado como una actividad fundamental para el ser humano, que sin embargo, presenta múltiples desafíos y dimensiones relevantes entre las que se encuentra la salud laboral. De acuerdo con la OIT (15) éste hace parte de la dignidad humana, en donde se deben fomentar condiciones decentes que lleven a un adecuado y óptimo bienestar psicológico.

Dentro de los parámetros de la perspectiva mundial ésta investigación tiene como meta aportar conocimientos al diagnóstico específico sobre la salud mental en educación superior. Desde esta perspectiva, se evidencia la necesidad de políticas preventivas que mitiguen las barreras percibidas por el personal administrativo; igualmente, apoya el acrecentamiento de motivadores y claves para la acción a partir de modelos cognitivos, siendo las creencias una de las alternativas propicias para la predicción de los comportamientos saludables (16).

En relación con el factor protector, el personal administrativo cuenta con el apoyo social para enfrentar los problemas de la vida cotidiana. Soporte que se cimenta en la familia y la pareja, al igual que en los hábitos y costumbres que ha aprendido y que le permiten manejar los conflictos de forma adecuada y adaptativa. En este sentido, aprende a darle importancia a la motivación en el trabajo y a combinarla con el potencial de creencias personales sobre su sentido en la organización.

Por otra parte, la mayoría de los administrativos perciben que están capacitados para afrontar las situaciones difíciles de la vida cotidiana; también, consideran que tienen la posibilidad de disminuir los riesgos, al practicar comportamientos saludables para su desarrollo personal y social, relacionados con: la asistencia a profesionales de la salud mental, la búsqueda de estrategias para obtener los servicios y el compromiso con dichos espacios.

Igualmente, los entrevistados consideran como factores protectores dentro de sus creencias y representaciones mentales su percepción sobre el beneficio de los avances en salud mental y la disponibilidad para seguir recomendaciones y adherirse a tratamientos; además, reconocen la importancia de la relación especialista - paciente.
Volviendo la mirada de nuevo, hacia los factores protectores sociales, el personal administrativo reconoce la importancia que tienen los medios de comunicación en la divulgación y socialización de los cuidados; también, creen que existe un clima adecuado para su desarrollo en su medio laboral.

Si bien es cierto que se identifican múltiples factores positivos que motivan y que consolidan las claves para el cambio hacia una conducta saludable, es importante señalar que desde la mitigación del riesgo, se debe centrar la atención en el factor estrés que es percibido como el fenómeno que afecta en un $65 \%$ la salud mental de los trabajadores. Como síntoma fundamental se presenta un inadecuado manejo del estrés, que se manifiesta en los estados de ánimo, los problemas de relación con la pareja y la comunicación inadecuada.

Las evidencias anteriores, invitan a reflexionar sobre la importancia de disponer de unas adecuadas condiciones de trabajo, a fin de evitar que se afecte la salud laboral, el desempeño y calidad en el trabajo, disminuyendo la posibilidad de desencadenar trastornos tanto físicos como psicológicos, accidentalidad y hasta la muerte. De acuerdo a estudios recientes del Ministerio de Trabajo en Argentina (17) se debe dar importancia a las acciones preventivas teniendo en cuenta la ergonomía, el tipo de trabajo, las jornadas laborales y las características del día a día.

Se debe precisar, que desde de la Psicología de la salud los factores organizacionales del trabajo como: las jornadas, el tiempo de descanso y su distribución, el ritmo, los tipos de control, la variedad del trabajo y la remuneración; al igual que las condiciones individuales como: la edad, género, rasgo y tipo de personalidad y aspectos familiares, deben considerarse como moduladores que potencializan o minimizan el riesgo de aparición de desórdenes de salud mental (18).

De manera análoga, se debe tener en cuenta que una de las percepciones de gravedad se aprecia en las preocupaciones que producen ansiedad, generando dificultades en la comunicación; aspecto que ha sido identificado como riesgo psicosocial en la salud mental en otros estudios $(19,20)$.

En este sentido es fundamental pensar en que la Universidad como una organización saludable y resilente, debe integrar tres elementos claves: 1) prác- 
ISSN-PRINT

1794-9831

E-ISSN 2322-7028

Vol. 15 No. 2

Jul - Dic 2018

Cúcuta, Colombia ticas y recursos organizacionales saludables (apoyo social y estrategias organizacionales saludables); 2) empleados saludables (creencias sobre su eficacia, afecto positivo y engagement); $y, 3$ ) resultados organizacionales saludables (desempeño, calidad y compromiso) (21).

Por ello se hace necesario e imperativo contar con alianzas y la cooperación de los servicios asistenciales locales y regionales (atención primaria especializada), lo que facilitará la oferta de servicios sanitarios disponibles, estableciendo un nivel de respuesta óptimo a la demanda de los mismos; factores que permitirán desarrollar técnicas de detección temprana e intervenciones precoces para prevenir o retrasar la progresión de la enfermedad (22).

Si bien es cierto que la familia es la red de apoyo principal, también representa un espacio donde se desarrollan frecuentemente situaciones de pérdida de control, especialmente en lo relacionado con el manejo inadecuado de situaciones laborales que recargan las responsabilidades; razón por la cual es importante trabajar este aspecto en las políticas de desarrollo laboral de las organizaciones (23).

Vale la pena reseñar que la OMS (24) reconoció la importancia de saber disfrutar de una vida laboral saludable y su manejo en el día a día, considerándola como un aspecto fundamental que debe ser trabajado. Al respecto, en el diagnóstico que realizó Colombia en el 2015 se comprobó que los riesgos que afectan la salud mental de los trabajadores se relacionan con: el manejo inadecuado del estrés, inestabilidad afectiva especialmente en lo relacionado con estados de depresión y aumento de los niveles de ansiedad; factores que están directamente relacionados con las incongruencias entre el trabajo a realizar y las competencias que se tienen, las limitaciones de tiempo para ejecutar los objetivos propuestos, el no contar con un equipo de trabajo, la ineficacia de los programas de incentivos y los problemas de comunicación en el trabajo.

Se quiere resaltar, que los resultados de esta investigación proporcionarán herramientas de trabajo útil en el área de control de impulsos y toma de decisiones. Es por eso que se sugiere trabajar de forma general con la población ampliando el repertorio de estrategias para enfrentar situaciones diarias de la vida, puesto que estudios como el de
Ibarra (25) demuestran la importancia que tiene para las organizaciones que sus empleados sientan la necesidad de autocontrol en situaciones de estrés y manejen con inteligencia emocional todas aquellas situaciones que desbordan sus recursos personales.

Por otra parte, se debe enfatizar en que existen casos en el personal administrativo por los que se ve afectado tales como: pérdida de peso, alteraciones y/o malestar de colón, gastritis, dolor de espalda, de cuello, falta de apetito y alteraciones físicas relacionadas con aspectos de la salud mental; por lo que se sugiere pensar en la necesidad de instaurar una cultura saludable en la universidad, con consciencia crítica sobre la responsabilidad individual y organizacional frente al logro de cambios en los estilos de vida (26).

También, se contempla la salud mental como un área en la que convergen diferentes aspectos a los cuales es necesario prestar atención, y por tanto desarrollar elementos que permitan obtener beneficio y calidad de vida afirmando lo expuesto por Gómez et al. (27) respecto a la encuesta de salud mental en Colombia.

De modo similar, se sugiere tener en cuenta los lineamientos del Ministerio de Salud y Protección Social para los entornos laborales saludables (28), relacionados con una intervención que no sólo se centre en los síntomas expresados por el personal administrativo, sino también en los antecedentes o agentes estresantes, moduladores y consecuentes que puedan variar tanto a nivel personal, como interpersonal y organizacional.

Así mismo, en este estudio se identifican los factores protectores percibidos por el personal administrativo desde su pensar, sentiry actuar, resaltando su capacidad y habilidad para desarrollarse humanamente; igualmente, se reconoce la importancia de trabajar aspectos para mejorar la calidad de vida laboral, así como lo plantean García et al (29) para quienes son fundamentales: el bienestar, la satisfacción, la seguridad, la identificación y la integración al puesto de trabajo.

Por tanto, es importante desarrollar planes, programas y proyectos de promoción, de difusión y psicoeducación con el propósito de desarrollar la conciencia y la cultura en salud mental, tal como lo afirman Romano y Scott (30) para quienes los programas de promoción de la salud deben incorporar el Modelo de 
Creencias en Salud, para asegurar la adherencia de los participantes al programa de adaptación de estilos de vida saludables.

Aunque existen diversidad de opiniones en cuanto al impacto del consumo de sustancias psicoactivas en el contexto de la universidad saludable, es importante instaurar una política de prevención de la farmacodependencia que no solo incluya las sustancias psicoactivas ilegales, sino también con mayor especificidad las sustancias psicoactivas legales como el cigarrillo, el alcohol y los medicamentos para el tratamiento de alguna enfermedad psicológica, entre otros; teniendo en cuenta lo referenciado por Renuka y Pushpanjali (31) sobre el modelo de creencias en salud y su efectividad en el tratamiento y la prevención del tabaquismo.

Es importante resaltar que el personal administrativo está motivado a desarrollar acciones en beneficio de su salud mental, entre las cuales se pueden destacar: disfrute y bienestar, autoestima, afectivo emocionales, fortalecimiento de la comunicación y toma de decisiones, entre otras.

Es por eso, que la salud mental para el personal administrativo tiene una importancia elevada, por tanto se requiere de estrategias y de ayudas externas que faciliten su consecución. También, se debe tener en cuenta que es un indicador que necesita de los servicios en salud mental para mantener y promover en los sitios de trabajo el bienestar y la calidad de vida.

Para concluir, se puede decir que en el caso específico de esta universidad, el personal administrativo percibe el ambiente laboral como un mantenedor de la salud mental; situación que se convierte en un factor favorable para implementar y seguir consolidando este espacio como un escenario que facilite el desarrollo de estrategias y de acciones en pro del bienestar emocional, psicológico y social.

Por tanto, se sugiere desplegar acciones que promuevan los espacios laborales como escenarios que fortalezcan y permitan fomentar una salud mental positiva, mediante el incremento en los encuentros, los servicios, las actualizaciones y capacitaciones, de tal modo que permitan visibilizar de forma general y continua el ambiente laboral como un escenario que mantenga siempre una salud mental positiva.

\section{Conclusiones}

- La universidad como constructora de dinámicas de conocimiento debe incentivar la inteligencia del bien común, respondiendo al desafío de la salud mental laboral, promoviendo y acrecentando el bienestar físico, mental y social de todos sus integrantes, asegurando las óptimas condiciones de trabajo.

- Es fundamental tener en cuenta el aporte del modelo de creencias en salud propuesto por autores funcionalistas como: Rosenstock, Becker, Janz, Maiman, Hochbaum, y Kegeles. Desde esta perspectiva, se debe partir de las necesidades de educación para la salud, que expliquen las conductas sobre las estructuras cognitivas con una postura personalizada, centrada en las dimensiones favorecedoras de la salud.

- El cambio conductual sobre el modelo permite creer que se puede producir una transformación favorable desde la posibilidad de percibir amenazas; lo que significa conocer la susceptibilidad en la que se encuentra, ser consciente de la medición propia de la severidad, los beneficios y barreras frente a la conducta posible, al igual que las motivaciones y las claves para la acción, dando cuenta de la eficiencia que se puede tener generando factores protectores que mitigan los riesgos (14) (32).

- El modelo de creencias en su dimensión susceptibilidad percibida, sirvió para identificar que los trabajadores de la entidad de educación superior perciben como riesgos y/o amenazas el no tener programas que involucren sus sistemas familiares; de ahí que se deban trabajar los aspectos de desarrollo humano individual en temas como la ansiedad y el manejo del estrés en la vida cotidiana.

- Las barreras se centran en la dificultad que perciben los administrativos ante la ausencia de tiempo para desarrollar un estilo de vida saludable y para mantener una salud mental positiva.

- En cuanto a las motivaciones se concluye que es importante creer que cualquier factor de riesgo puede disminuirse y/o atenuarse a través del desarrollo de comportamientos saludables y actividades en general, entre las que se pueden 
ISSN-PRINT

1794-9831

E-ISSN 2322-7028

Vol. 15 No. 2

Jul - Dic 2018

Cúcuta, Colombia destacar: disfrute y bienestar, autoestima, afectivo - emocionales, fortalecimiento de la comunicación, toma de decisiones, crecimiento espiritual y actividad física, entre otras.

- Como una clave para la acción, se percibe como importante mantener un ambiente de trabajo agradable que facilite la consolidación de la salud mental; factor que puede ser de utilidad para generar mejores espacios, capacitaciones, encuentros, servicios que permitan mantener esta percepción.

\section{Conflicto de Intereses}

Las autoras declaran no tener ningún conflicto de intereses.

\section{Referencias Bibliográficas}

1. Garrido Larrea P, Ansoleaga Moreno E, Tomicic Suñer A, Domínguez Valverde C, Castillo Vergara S, et al. Afecciones de Salud Mental y el Proceso de Retorno al Trabajo: Una Revisión Sistemática. Cienc. Trab. [Internet]. 2013 [consultado 2018 Abr 05]; 15(48):105-113. Disponible en: https:// scielo.conicyt.cl/scielo.php?script $=$ sci arttext\&pid $=$ S0718-24492013000300002\&lng=es

2. Caraveo J, Calatayud A, López S. Evaluación de la salud mental ocupacional. Salud Pública de México [Internet]. 2014 [consultado 2018 Abr 05]; 27(5):391-401. Disponible en: http://saludpublica.mx/ index.php/spm/article/view/418

3. Vieco Gómez GF, Abello Llanos R. Factores psicosociales de origen laboral, estrés y morbilidad en el mundo. Psicología desde el Caribe [Internet]. 2014 [consultado 2018 Abr 23]; 31(2): 354 - 385. Recuperado de: http://www.redalyc.org/articulo.oa?id=21331836004

4. Corchuelo M, Soler M, Lozano L. Informe ejecutivo de la II encuesta nacional de condiciones de seguridad y salud en el trabajo en el sistema general de riesgos laborales. Ministerio del Trabajo. Informe Ejecutivo de la Segunda Encuesta Nacional de Condiciones de Seguridad y Salud en el Trabajo en el Sistema General de Riesgos Laborales de Colombia. Bogotá; 2013.

5. Bernardo MH, Souza HA, Garrido Pinzón J, Kawamura EA. Salud mental relacionada con el trabajo: desafíos para las políticas públicas. Universitas Psychologica [Internet]. 2015 [consultado 2018 Abr 23]; 14(5):1613-1624. Disponible en: http://dx.doi.org/10.11144/Javeriana.upsy14-5.smrt

6. Ureña Bonilla P, Barrantes Brais K, Solís Bastos L. Bienestar psicológico, espiritualidad en el trabajo y percepción subjetiva de la salud en personal académico y administrativo de la Universidad Nacional. Revista Electrónica Educare [Internet]. 2014 [consultado 2017 Sep. 26]; 18(1):155-175. Disponible en: http:// www.scielo.sa.cr/scielo.php?script $=$ sci arttext\&pid=S1409-42582014000100008\&lng=en\&tlng=es

7. Moccia, S. Felicidad en el trabajo. Papeles del Psicólogo [Internet]. 2016 [consultado 2017 Sep. 26]; 37(2):143-151. Recuperado de: http://www.redalyc.org/articulo.oa?id=77846055007

8. Ordoñez AC, Gómez E, Calvo A. Desórdenes músculo esqueléticos relacionados con el trabajo. Revista Colombiana de Salud Ocupacional, 6(1) 2016: 24-30

9. Tobón Lotero JD. La salud mental en Colombia. Un campo atravesado por la ley. CES Psicol [Internet]. 2013 [Consultado 05 Abril 2018]; 6(2):1-3. Disponible en: http://www.scielo.org.co/scielo. php?script $=$ sci_arttext\&pid=S2011-30802013000200001\&lng=en.

10. Mogollón Canal OM, Villamizar Carrillo DJ, Padilla Sarmiento SL. Salud mental en la educación superior: una mirada desde la salud pública. Rev.cienc.ciudad.2016; 13(1): 103-120.

11. Villamizar Carrillo DJ, Padilla Sarmiento SL, Mogollón Canal OM. Contexto de la salud mental en docentes universitarios. Un aporte a la salud pública. CES Salud Pública. 2015; 6:146-159.

12. Calderón C, Carrete L, Vera J. Validación de una escala de creencias de salud sobre diabetes tipo 1 para el contexto mexicano: HBM-T1DM. Salud Pública de México [Internet]. 2018 [Consultado 05 abril 2018]; 60(2):175-183. Disponible en: http://www.saludpublica.mx/index.php/spm/article/view/8838/11458.

13. Villalba Cuéllar JC, González Serrano A. La incidencia del modelo de educación superior y la intervención estatal en la calidad de la educación superior en Colombia. Prolegómenos. 2016; 19(37).

14. San Pedro EM, Roales JG. El modelo de creencias de salud: Revisión teórica, consideración crítica y propuesta alternativa. I Hacia un análisis funcional de las creencias en salud. Revista internacional de 
Psicología y terapia psicológica. 2003; 3(1):91-109.

15. Organización Internacional del Trabajo. Trabajo decente [Internet]. 2016 [consultado 4 de abril de 2018]. Disponible en: http://www.ilo.org/global/topics/decent-work/lang--es/index.htm

16. Flórez Alarcón L, Vélez Botero H, Rojas Russell M. Intervención motivacional en psicología de la salud: revisión de sus fundamentos conceptuales, definición, evolución y estado actual. Psychologia: avances de la disciplina. 2014; 8(2):49-71.

17. República de Argentina. Ministerio de Trabajo, empleo y seguridad social. Salud y seguridad en el trabajo (SST), aportes para una cultura de la prevención. 2014.

18. Ordóñez Hernández C, Mosquera Silva C, Patiño Segura J, Rebolledo Ángel F. Salud Mental de Trabajadores de Dos Instituciones de Educación Superior de Cali, Colombia. Revista Colombiana De Salud Ocupacional, 2015; 4(3):21-25.

19. Gutiérrez Strauss A, Viloria Doria J. Riesgo psicosociales y estrés en ambiente laboral. Revista Científica Salud Uninorte [Internet]. 2014 [consultado 2018 Abr 05]; 30(1):5-7. Disponible en: http:// rcientificas.uninorte.edu.co/index.php/salud/article/view/6411

20. Ruiz Torres CJ. El estrés y su relación en el desempeño laboral en la sede UGEL No =3. [Tesis]. Perú: Universidad César Vallejo. 2017 [consultado 2018 Abr 04] Disponible en: http://repositorio.ucv.edu. pe/handle/UCV/10025

21. Hernández Vargas CI, Llorens Gumbau S, Rodríguez Sánchez AM. Empleados saludables y calidad de servicio en el sector sanitario. Anales de Psicología [Internet]. 2014 [Consultado 28 de septiembre de 2017]; 30(1):237-248. Disponible en: http://www.redalyc.org/articulo.oa?id=16729452026

22. Irarrázaval $M$, Prieto $F$, Armijo J. Prevención e intervenciones tempranas en salud mental: una perspectiva internacional. Acta bioeth. [Internet]. 2016 [consultado 2018 Abr 04]; 22(1):37-50. Disponible en: http://dx.doi.org/10.4067/S1726-569X2016000100005

23. Gómez Vélez M, Calderón Carrascal P. Salud mental en el trabajo: entre el sufrimiento en el trabajo y la organización saludable. Katharsis. 2017; (23):17

24. Organización mundial de la Salud. Salud mental en el lugar de trabajo. [Internet] Ginebra; Octubre, 2017 [consultado 4 de abril de 2018]. Disponible en: http://www.who.int/mental health/in the workplace/es/

25. Ibarra LM. Inteligencia emocional desde la perspectiva empresarial. Revista Apuntes deAdministración. 2016; 1(1):76-80.

26. Sas Nowosielski K, Hadzik A, Górna J, Grabara M. Applying the health belief model in explaining the stages of exercise change in older adults. Polish journal of sport and tourism. 2016; 23(4).

27. Gómez Restrepo C, De Santacruz C, Rodríguez MN, Rodríguez V, Tamayo Martínez N, et al. Encuesta Nacional de Salud Mental Colombia 2015. Protocolo del estudio, Revista Colombiana de Psiquiatría. 2016; 45(1):2-8.

28. Ministerio de Salud y Protección Social. Entorno laboral saludable. Dirección de desarrollo y talento humano en salud. [Internet] Bogotá; 2016 [consultado 5 de abril de 2018]. Disponible en: https://www. minsalud.gov.co/sites/rid/Lists/BibliotecaDigital/RIDE/VS/TH/entorno-laboral-saludable-incentivoths-final.pdf

29. García Pazmiño MA, González Baltazar R, Aldrete Rodríguez MG, Acosta Fernández M, León Cortés SG. Relación entre Calidad de Vida en el Trabajo y Síntomas de Estrés en el Personal Administrativo Universitario. Cienc Trab. [Internet]. 2014 [consultado 2018 Abr 06]; 16(50):97-12. Disponible en: https://scielo.conicyt.cl/scielo.php?script=sci arttext\&pid=S0718-24492014000200007\&lng=es

30. Romano V, Scott I. Using Health Belief Model to reduce obesity amongst African American and Hispanic populations. Procedia - Social and behavioral sciences. 2014; 159:707-711.

31. Renuka P, Pushpanjali K. Effectiveness of Health Belief Model in Motivating for Tobacco Cessation and to Improving Knowledge, attitude and Behavior of Tobacco Users. Cancer and Oncology Research. 2014; 2(4): 43-50.

32. Nieto J, Abad M, Esteban A, Arreal T, Martínez J. Psicología para ciencias de la salud: Estudio del comportamiento humano ante la enfermedad. La dimensión social. México. 2015; 1-18. 